\title{
Continuous maps in terms of new convergence conditions
}

\author{
Asha Gupta \\ Department of Applied Sciences, PEC University of Technology, Chandigarh, India \\ Email: ashagoel30@yahoo.co.in
}

Copyright (C)2014 Asha Gupta. This is an open access article distributed under the Creative Commons Attribution License Creative Commons Attribution License, which permits unrestricted use, distribution, and reproduction in any medium, provided the original work is properly cited.

\begin{abstract}
It is well known that if $X$ is Frechet, then a map $f: X \rightarrow Y$ is continuous if and only if $\left\{x_{n}\right\} \rightarrow x$ in $X$ implies $\left\{f\left(x_{n}\right)\right\} \rightarrow f(x)$ in $Y$. In this paper, some new convergence conditions in terms of sequences have been introduced and in terms of these convergence conditions, generalisations and analogues of some known results of continuous maps are obtained.
\end{abstract}

Keywords: Convergence, continuous, Frechet, sequence.

\section{Introduction}

By a space, we shall mean a topological space. No separation axioms are assumed and no map is assumed to be continuous or onto unless mentioned explicitly; $\operatorname{cl}(A)$ will denote the closure of the subset $A$ in the space $X$. If $A$ is a subset of $X$, we say that $X$ is $T_{1}$ at $A$ if each point of $A$ is closed in $X . X$ is said to be Frechet space (or closure sequential in the terminology of Wilansky [1]) if for each subset $A$ of $X, x \in c l(A)$ implies there exists a sequence $\left\{x_{n}\right\}$ in $A$ converging to $x$. $X$ is said to be a $k$-space if $O$ is open (equivalently :closed) in $X$ whenever $O \cap K$ is open (closed) in $K$ for every compact subset $K$ of $X$. Every space which is either locally compact or Frechet is a $k$-space. A map $f: X \rightarrow Y$ is said to be compact preserving if image of each compact set is compact.

In 1968,R.V. Fuller [2] proved the following theorem for topological spaces $X$ and $Y$

Theorem 1.1 (2,Theorem 3.4) . Let $f: X \rightarrow Y$ be a map. A sufficient condition that $f$ be continuous is that $f$ have a closed graph and be subcontinuous. If $Y$ is $T_{2}$, the condition is necessary also.

In 1975, N.Liden [3] has proved the following theorem

Theorem 1.2 (3,Theorem 4). Let $Y$ be $T_{2}$ and $X$ be a $T_{2}, k$-space. Then $f: X \rightarrow Y$ is continuous if and only if it is compact-preserving and has closed fibers.

It is well known that if $X$ is Frechet, then a map $f: X \rightarrow Y$ is continuous if and only if $\left\{x_{n}\right\} \rightarrow x$ in $X$ implies $\left\{f\left(x_{n}\right)\right\} \rightarrow f(x)$ in $Y$. Characterizations of continuous maps in terms of convergence of sequences have been 
given by Garg and Goel [see4,5,6].In this paper, we introduce new convergence conditions $S(a)-S(d)$ in terms of sequences and give analogues and generalizations of the above results of Fuller and Liden.

\section{Convergence conditions}

For any map $f: X \rightarrow Y$, we consider the following convergence conditions $S(a)-S(d)$.

$S(a):\left\{x_{n}\right\} \rightarrow x$ implies $\left\{f\left(x_{n}\right)\right\} \rightarrow f(x)$.

$S(b): x$ is a cluster point of $\left\{x_{n}\right\}$ implies $f(x)$ is a cluster point of $\left\{f\left(x_{n}\right)\right\}$

$S(c)$ : Whenever $\left\{x_{n}\right\}$ has a cluster point, say $x,\left\{f\left(x_{n}\right)\right\}$ has a cluster point $y$, and if $y$ is the only cluster point of $\left\{f\left(x_{n}\right)\right\}$, then $y=f(x)$.

$S(d)$ : Whenever $\left\{x_{n}\right\}$ has a cluster point, $\left\{f\left(x_{n}\right)\right\}$ has a cluster point.

Remark 2.1. It is well known that if $f: X \rightarrow Y$ is continuous, then condition $S(a)$ holds. Converse holds if $X$ is Frechet.

Remark 2.2. Obviously continuity $\Rightarrow S(b) \Rightarrow S(c) \Rightarrow S(d)$.

Theorem 2.1 $S(b) \Rightarrow S(a)$ but not conversely. The converse holds if $X$ is Frechet.

Proof. Suppose $f: X \rightarrow Y$ and $\left\{x_{n}\right\} \rightarrow x$. If $\left\{f\left(x_{n}\right)\right\}$ does not converge to $f(x)$ then there exists a neighbourhood $V$ of $f(x)$ such that $f\left(x_{n}\right)$ is not eventually in $V$. This means there exists a subsequence $\left\{y_{n}\right\}$ of $\left\{x_{n}\right\}$ such that $f(x)$ is not a cluster point of $\left\{f\left(y_{n}\right)\right\}$, a contradiction to $S(b)$, since $x$ is a cluster point of $\left\{y_{n}\right\}$. For the converse, if $X$ is Frechet, $S(a)$ implies continuity of $f$ and hence $S(b)$ holds.

The following example shows that $S(a)$ does not imply even $S(d)$, in general.

Example 2.2 For any $t \in \beta N-N$, let $X=N \cup\{t\}$ as a subspace of $\beta N$ and let $Y$ be the set $X$ with discrete topology. Since there is no non trivial convergent sequence in $X$, the identity map $i: X \rightarrow Y$ satisfies $S(a)$. To prove $i$ does not satisfy even $S(d)$, consider the sequence $\left\{x_{n}\right\}$ in $X$ where $x_{n}=n$ for each $n$ in $N$. Obviously $t$ is a cluster point of $\left\{x_{n}\right\}$ in $X$, but the sequence $\left\{x_{n}\right\}$ has no cluster point in $Y$.

\section{Continuous maps}

We now give characterizations of continuous maps in terms of the convergence conditions $S(b)-S(d)$ under some conditions on $X, Y$ and $f$.

Theorem 3.1 Let $f: X \rightarrow Y$ be any map, where $X$ is Frechet. Then $f$ is continuous if and only if condition $S(b)$ holds.

Proof. The proof follows from theorem 2.1 and remark 2.1 above.

For the proof of the following theorem 3.5, we need the following

Lemma 3.2 Let $X$ be a Frechet space. A point $x$ of $X$ is a cluster point of a sequence of points $\left\{x_{n}\right\}$ in $X$ if and only if there exists a subsequence of $\left\{x_{n}\right\}$ which converges to $x$ (no separation axiom assumed).

Proof. Let $x$ be a cluster point of the sequence $\left\{x_{n}\right\}$ in $X$ and let $A$ be the range of the sequence $\left\{x_{n}\right\}$. Now $x \in \operatorname{cl}(A)$, since $\left\{x_{n}\right\}$ clusters at $x$. Then $X$ is Frechet implies that there exists a sequence of points in $A$ converging to $x$. Let $D$ be the set of all sequences of distinct points of $A$ converging to $x$ and $C$ be the set of all constant sequences of points of $A$ converging to $x$. 
In case $D \neq \phi$, there exists a sequence of distinct points of $A$ which can be rearranged, if necessary, to obtain a subsequence of $\left\{x_{n}\right\}$ converging to $x$.

In case $D=\phi$, we have $C \neq \phi$. If $C$ is infinite, then there exist infinitely many constant sequences of points in $A$ converging to $x$ and therefore, there exists a sequence of distinct points of $A$ which can be rearranged, if necessary, to obtain a subsequence of $\left\{x_{n}\right\}$ converging to $x$. If $C$ is finite, suppose $C=\left\{\left\{t_{1}\right\},\left\{t_{2}\right\}, \ldots . .\left\{t_{k}\right\}\right\}$, where each $\left\{t_{i}\right\}$ is a constant sequence in $A$ converging to $x$. If no constant sequence $\left\{t_{i}\right\}$ is a subsequence of $\left\{x_{n}\right\}$, then $A \backslash\left\{t_{1}, t_{2}, \ldots . . t_{k}\right\} \neq \phi$ and since $x$ is a cluster point of the sequence $\left\{x_{n}\right\}$, it follows that $x \in \operatorname{cl}\left(A \backslash\left\{t_{1}, t_{2}, \ldots . . t_{k}\right\}\right)$. Then $X$ is Frechet implies there exists a sequence $\left\{a_{n}\right\}$ in $A \backslash\left\{t_{1}, t_{2}, \ldots . . t_{k}\right\}$ such that $a_{n} \rightarrow x$. Since $a_{j} \in$ $A \backslash\left\{t_{1}, t_{2}, \ldots . . t_{k}\right\}$ for each $j$, no point of sequence $\left\{a_{n}\right\}$ can be repeated infinitely often in the sequence $\left\{a_{n}\right\}$. Therefore, from $\left\{a_{n}\right\}$, we can obtain a sequence of distinct points of $A$ which converges to $x$. This is impossible, since $D=\phi$. Therefore, at least one $\left\{t_{i}\right\}$ is a constant subsequence of $\left\{x_{n}\right\}$ which converges to $x$, in case $C$ is finite. Converse is obvious for any space $X$.

Corollary 3.3 A Frechet space is sequentially compact if and only if it is countably compact.

Since Frechet is hereditary, from corollary 3.3 follows immediately the following

Corollary 3.4 A subset of a Frechet space is sequentially compact if and only if it is countably compact.

Theorem 3.5 Let $f: X \rightarrow Y$ be any map where $X$ is Frechet and $Y$ is Frechet, $T_{2}$. Then $f$ is continuous if and only if condition $S(c)$ holds.

Proof. Necessity of the condition is obvious for arbitrary $X$ and $Y$. For the sufficiency, we prove condition $S(b)$ holds. Suppose $\left\{x_{n}\right\}$ is any sequence of points in $X$ with a cluster point $x$. Then by condition $S(c)$, the sequence $\left\{f\left(x_{n}\right)\right\}$ has a cluster point, say $y$ in $Y$. Since $Y$ is Frechet, there exists a subsequence $\left\{f\left(y_{n}\right)\right\}$ of $\left\{f\left(x_{n}\right)\right\}$ such that $f\left(y_{n}\right) \rightarrow y$ by lemma 3.2. Now $\left\{y_{n}\right\}$, being a subsequence of $\left\{x_{n}\right\}$ converges to $x$ and therefore, $x$ is a cluster point of $\left\{y_{n}\right\}$. Moreover, $y$ is the only cluster point of $\left\{f\left(y_{n}\right)\right\}$, since $Y$ is $T_{2}$. Therefore, by condition $S(c), y=f(x)$ and so $f(x)$ is a cluster point of $\left\{f\left(x_{n}\right)\right\}$. Thus condition $S(b)$ holds and $\mathrm{f}$ is continuous by theorem 3.1 .

Theorem 3.6 Let $f: X \rightarrow Y$ be any map with closed fibers, where $X$ is a $T_{2}, k$-space and $Y$ is either second countable and $T_{2}$ or metrizable. Then $f$ is continuous if and only if condition $S(c)$ holds.

Proof. The proof follows from the following lemma 3.7 and theorem 1.2 of Liden of section 1.

Lemma 3.7 Let $Y$ be either second countable and $T_{2}$ or metrizable. A map $f: X \rightarrow Y$ is compact-preserving if condition $S(c)$ holds.

Proof. Let $A$ be a compact subset of $X$ and $\left\{f\left(x_{n}\right)\right\}$ be any sequence of points in $f(A)$, where $x_{n} \in A$ for each $n$. Now $\left\{x_{n}\right\}$, being a sequence of points in the compact set $A$, has a cluster point. Then by condition $S(c)$, the sequence $\left\{f\left(x_{n}\right)\right\}$ has a cluster point, say $y$ and therefore by lemma $3.2,\left\{f\left(x_{n}\right)\right\}$ has a subsequence $\left\{f\left(y_{n}\right)\right\}$ converging to $y$, since $Y$ is Frechet. Now $Y$ is $T_{2}$ implies $y$ is the only cluster point of $\left\{f\left(y_{n}\right)\right\}$ and since $A$ is compact, the sequence $\left\{y_{n}\right\}$ in $A$ has a cluster point, say $x$ in $A$. Therefore, by condition $S(c), y=f(x) \in f(A)$. Hence $f(A)$ is countably compact and therefore compact, since $Y$ is either second countable or metrizable.

Theorem 3.8 Let $f: X \rightarrow Y$ be any closed map with closed fibers and either

(a) $X$ is a Frechet space which is either $T_{2}$ or regular or normal or

(b) $X$ is a $T_{2}, k$-space and $Y$ is either second countable and $T_{2}$ or metrizable.

Then $f$ is continuous if and only if condition $S(d)$ holds.

Proof. Necessity of the condition is obvious for arbitrary spaces $X$ and $Y$. For the sufficiency, first suppose $(a)$ holds. As in the proof of theorem 3.5, we prove $S(b)$ holds. If $\left\{x_{n}\right\}$ is any sequence of points in $X$ with a cluster point $x$, then since $X$ is Frechet by lemma 3.2. there exists a subsequence $\left\{y_{n}\right\}$ of $\left\{x_{n}\right\}$ such that $y_{n} \rightarrow x$. Then condition $S(d)$ implies $\left\{f\left(y_{n}\right)\right\}$ has a cluster point, say $y$. If $y \neq f(x)$, then the set $U=X-f^{-1}(y)$ is an open set 
containing the closed set $f^{-1}(f(x))$ so the point $x$.

First consider the case when $X$ is $T_{2}$. Since $y_{n} \rightarrow x$, there exists a positive integer $n_{0}$ such that $y_{n} \in U$ for all $n \geq n_{0}$. If $K=\left\{y_{n}: n \geq n_{0}\right\} \cup\{x\}$, then $K$ is compact and closed, since $X$ is $T_{2}$ and so $f(K)$ is closed, which is a contradiction since $y \in \operatorname{cl} f(K)-f(K)$. Therefore, $y=f(x)$ and so $f(x)$ is a cluster point of $\left\{f\left(x_{n}\right)\right\}$. Thus condition $S(b)$ holds and hence $f$ is continuous in this case.

In case $X$ is regular or normal, there exists an open set $V$ containing $x$ such that $c l V \subset U$, since $f^{-1}(f(x))$ is a closed set contained in $U$. Since $y_{n} \rightarrow x$ there exists a positive integer $n_{0}$ such that $y_{n} \in V$ for all $n \geq n_{0}$ and therefore $f\left(y_{n}\right) \in f(c 1 V)$ for all $n \geq n_{0}$. Also $f(c 1 V)$ is closed and so $y \in f(c 1 V)$, which is a contradiction since $c l V \subset X-f^{-1}(y)$. Therefore, $y=f(x)$ and hence $f$ is continuous in this case also.

In case $(b)$ holds, continuity of $f$ follows from the following lemma 3.9 and theorem 1.2 Liden of section 1.

Lemma 3.9 Let $X$ be $T_{2}$ and $Y$ be either second countable or metrizable. A closed map $f: X \rightarrow Y$ is compactpreserving if condition $S(d)$ holds.

Proof. Let $A$ be a compact subset of $X$ and $\left\{f\left(x_{n}\right)\right\}$ be any sequence of points in $f(A)$, where $x_{n} \in A$ for each $n$. Now $\left\{x_{n}\right\}$, being a sequence in the compact set $A$, has a cluster point. Then by condition $S(d)$, the sequence $\left\{f\left(x_{n}\right)\right\}$ has a cluster point (in $f(A)$, since the set $f(A)$ is closed), implying thereby that $f(A)$ is countably compact and hence compact since $Y$ is either second countable or metrizable. Hence, $f$ is compact-preserving.

\section{Conclusion}

In this paper, characterizations of continuous maps in terms of new convergence conditions of sequences have been obtained.

\section{References}

[1] A. Wilansky, Topology for analysis, Xerox College Publishing Lexington, Massachusetts, Toronto,(1970).

[2] R. V. Fuller, Relations among continuous and various non continuous functions, Pacific J. Math., 25, (1968) $495-509$.

[3] N. Liden, K-Spaces,their anti spaces and related mappings, Pacific J. Math., 50, (1975), 505-514.

[4] G. L. Garg, A. Goel, Convergence and perfect maps in metric spaces, Indian Journal of Pure and Applied Mathematics, 27,7, (1996), 633-637.

[5] G. L.Garg, A. Goel, Some conditions implying continuity of Maps, Acta Math. Hungarica, 81,3, (1998), $271-274$.

[6] G. L.Garg, A. Goel, Continuity of Maps in terms of cluster points, International Journal Of Pure and Applied Mathematics, 51, (2009), 431-435. 